\title{
Cognitive behavioural therapy reduced symptoms in patients with obsessive-compulsive disorders with no overt compulsive rituals
}

Freeston MH, Ladouceur R, Gagnon F, et al. Cognitive-behavioral treatment of obsessive thoughts: a controlled study.J Consult Clin Psychol 1997Jun,65:405-13.

\section{Objective}

To determine the effectiveness of cognitive behavioural therapy for patients with obsessive-compulsive disorder (OCD) without overt compulsive rituals.

\section{Design}

Randomised controlled trial.

\section{Setting}

Quebec, Canada.

\section{Patients}

29 referred or self referred patients (mean age 36 y, 55\% men) who were diagnosed with OCD based on the Diagnostic and Statistical Manual of Mental Disorders, 3rd edition, revised; used cognitive neutralising activities; had no overt compulsions or compulsions functionally related to the target obsession; and did not meet diagnostic criteria for substance abuse disorder, psychotic disorders, organic mental disorder, paraphilia, or impulse control disorders and completed a pretreatment assessment. Patients with mood or anxiety disorders secondary to OCD, and those receiving medication at a stable dosage for $\geqslant 12$ weeks, were eligible.

\section{Intervention}

Patients allocated to treatment $(\mathrm{n}=15)$ met with trained therapists twice weekly for 1.5 hour sessions and received a standardised programme (a cognitive account of obsessions, training in exposure and response prevention, cognitive restructuring, and instruction in relapse prevention). Therapy termina- tion was based on clinical improvement or a maximum of 40 sessions. Follow up sessions were held at 1, 2, and 3 months, and a booster session occurred at 6 months. Patients allocated to the wait list group $(n=14)$ were told that therapy would begin 16 weeks after the initial assessment and were telephoned monthly.

\section{Main outcome measures}

Mean post-test scores on the Yale-Brown Obsessive-Compulsive Scale (Y-BOCS), Current Functioning Assessment (CFA), Padua Inventory (PI), Beck Anxiety Inventory (BAI), and Beck Depression Inventory (BDI).

\section{Main results}

Intention to treat analysis showed lower mean post-test scores (better functioning) for the treatment group compared with the wait list group on the Y-BOCS (12.2 v 22.0), CFA (4.7 v 5.7), PI $(54.7 v 83.9)$, and BAI $(12.8 v 21.6)(\mathrm{p}<0.05)$. No differences were seen for BDI scores. Treatment group mean post-test scores for all tests were lower than mean pre-test scores $(\mathrm{p}<0.001)$, but control group mean pre-test and post-test scores were not different.

\section{Conclusion}

Cognitive behavioural treatment reduced symptoms in patients with obsessive-compulsive disorders without overt compulsive rituals.

Sources of funding: Le Fonds de la Recherche en Santé du Québec (FRSQ) and Medical Research Council of Canada.

For article reprint: Dr M H Freeston, Centre de recherche Fernand-Seguin, 7331 rue Hochelaga, Montréal, Québec H1N 3V2, Canada. Fax +1 5142512617.

\section{Commentary}

The study by Freeston $e t$ al makes a major contribution to findings on OCD. While much has been achieved with the treatment of patients with OCD and overt compulsive rituals, the work with those with no overt compulsions has been limited. Interesting and important theoretical contributions have been made, but rigorous empirical work on the treatment of covert OCD phenomena has been conspicuous by its absence.

The study by Freeston et al undertook a systematic evaluation of a treatment package for this problem. The package was founded upon the following features of the problem: importance of the appraisal of the obsession; erroneous sense of responsibility; neutralising strategies that the patient uses to reduce the effects of the obsession; and the seeking of reassurance. The package consisted of elements specifically designed to deal with these features. This cognitive behavioural treat- ment package was shown to be effective. Compared with waiting list controls, the patients in the treatment group showed substantial improvement. Most of the gains were retained at follow up. The findings have implications for practice. Until now patients with OCD and no overt rituals have been seen as difficult to treat. The results of this trial will encourage clinicians to treat these patients, and give them a tested package.

One concern is that the treatment was long, with a mean of 40.5 hours. Such a large therapeutic input may be problematic in routine clinical settings. Freeston $e t$ $a l$ say that the relative inexperience of the therapists and the difficulties of applying a new approach may have been part of the reason for this large time requirement. When the package is retested and refined, more rapid improvement may be achieved. Work also needs to be done on the relative contributions of the various components of the package to outcome.

The recent paper by Rachman provides new ideas and insights which are likely to contribute to future research in this area, ${ }^{1}$ including the refinement of the study by Freeston et al.

The review by Abramowitz is a metaanalysis of the studies on psychological and pharmacological treatment of OCD. Because of the increase in controlled trials for the treatment of OCD and the claims made for the efficacy of SRI drugs with this patient group, a review of the field was needed.

Thirty two studies were included in this analysis. These included 37 treatment comparisons. The treatments were ERP, other psychological treatments, and SRI and non-SRI medication. The metaanalysis showed that ERP and cognitive therapy were effective in patients with OCD. The efficacy of ERP is well established, so this comes as no surprise. The (continued on page 13) 


\section{Review: exposure with response prevention and serotonergic medication reduce symptoms of obsessive-compulsive disorder}

Abramowitz J S. Effectiveness of psychological and pharmacological treatments for obsessive-compulsive disorder: a quantitative review. J Consult Clin Psychol 1997 Feb; 65:44-52.

\section{Objective}

To evaluate the effectiveness, using meta-analysis, of treatments for obsessive-compulsive disorder (OCD) and to determine if some treatments are more effective than others .

\section{Data sources}

Studies were identified by searching PsycLIT and Medline databases; by handsearching relevant journals; and by scanning the bibliographies of relevant studies. Only published research was considered for inclusion.

\section{Study selection}

Studies were selected if they were randomised controlled trials of adults with a primary diagnosis of OCD and if outcome measures of OCD symptoms were reported at post-treatment or follow up. Crossover designs were included only if outcomes were reported for each group separately before the point of crossover. Studies were excluded if patients had concurrent diagnoses of OCD together with active phases of other disorders, or if the investigators had failed to use explicit, standardised diagnostic criteria for OCD.

\section{Data extraction}

Data were extracted on number of patients included; patient characteristics including age, sex, and duration of OCD symptoms; type and length of treatment; number of professional contacts per week; drop out rate; and pretreatment and post-treatment OCD symptoms.

\begin{abstract}
Main results
52 studies met the selection criteria, but 20 investigated treatments appeared only once in the literature and were therefore excluded. The remaining 32 studies included a total of 37 treatment comparisons. A large effect favouring exposure with response prevention (ERP) was found in 2 comparisons with progressive muscle relaxation (effect size 1.18, p $<0.01$ ). ERP did not differ statistically from cognitive therapy or single components of ERP (exposure alone or response prevention alone). Serotonin reuptake inhibitors (SRIs) led to greater reductions in OCD symptoms than did placebo (effect size 0.71 for self ratings, $\mathrm{p}<0.05$; effect size 1.09 for clinician ratings, $\mathrm{p}<0.01$ ). Clomipramine had the greatest effect as measured by clinician ratings (effect size 1.31, $\mathrm{p}<0.01$ ). Non-SRIs did not differ statistically from placebo (effect size 0.20 for clinician ratings). Clomipramine led to greater reductions in OCD symptoms than did non-SRI drugs (effect size 0.70 for self-ratings, 0.37 for clinician ratings, $\mathrm{p}<0.05$ for both) but not other SRIs (effect size 0.15 ).
\end{abstract}

\section{Conclusion}

Exposure with response prevention and serotonergic medication both reduce obsessive-compulsive disorder symptoms.

Source of funding: no external funding.

For article reprint:Dr I S Abramowitz, Department of Psychology, The University of Memphis, Memphis,TN 38152,USA.email:abramowitz@auhs.edu. (commentary continued from page 12)

"cognitive" approaches were heterogenous and overlapped with ERP, making it necessary to wait for more systematic work. As for the effects of medication, the SRIs were shown to produce substantial improvement in patients. However, non-SRI drugs did no better than placebo. It also appeared that clomipramine might be marginally more effective than other SRIs, but further direct comparisons are needed.

For the practising clinician, the analysis confirms the role of psychological treatments and SRI medication for patients with OCD. However, the analysis does not answer the question of the relative efficacy of the 2 treatments. The literature does not contain enough information on this so far. The other remaining question is the value of combined treatments. Again, future work is awaited.

Clinicians who apply psychological treatments will be interested to know more about the nature of effective components of psychological interventions in OCD. The finding in this analysis that cognitive therapy was also effective, highlights the need to define and develop specific cognitive interventions and evaluate them. Given the very limited number of studies of cognitive therapy and the lack of refinement of the techniques and packages, the better established ERP approach remains the psychological treatment of choice for patients with OCD.

Padmal da Silva, MA, MPhil

Institute of Psychiatry

London, UK

1 Rachman S. A cognitive theory of obsessions. Behav Res Ther 1997;35:793-802.

\section{Editor's Note}

See also: Antidepressant drugs relieve symptoms of obsessive-compulsive disorder [abstract]. Evidence-Based Medicine 1996 Mar-Apr;1:82. Abstract of: Piccinelli M, Pini S, Bellantuono C, et al. Efficacy of drug treatment in obsessive-compulsive disorder. A meta-analytic review. Br J Psychiatry 1995 Apr;166:424-43. 